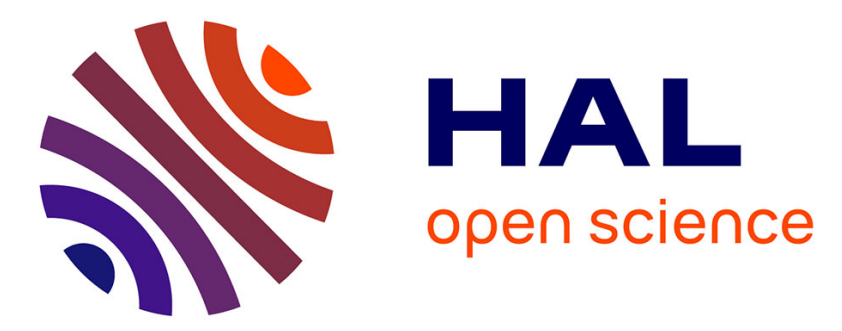

\title{
Analytical expression of elastic rods at equilibrium under 3D strong anchoring boundary conditions
}

\author{
Olivier Ameline, Sinan Haliyo, Xingxi Huang, Jean A.H. Cognet
}

\section{To cite this version:}

Olivier Ameline, Sinan Haliyo, Xingxi Huang, Jean A.H. Cognet. Analytical expression of elastic rods at equilibrium under 3D strong anchoring boundary conditions. Journal of Computational Physics, 2018, 373, pp.736 - 749. 10.1016/j.jcp.2018.07.021 . hal-01875301

\section{HAL Id: hal-01875301 \\ https://hal.sorbonne-universite.fr/hal-01875301}

Submitted on 17 Sep 2018

HAL is a multi-disciplinary open access archive for the deposit and dissemination of scientific research documents, whether they are published or not. The documents may come from teaching and research institutions in France or abroad, or from public or private research centers.
L'archive ouverte pluridisciplinaire HAL, est destinée au dépôt et à la diffusion de documents scientifiques de niveau recherche, publiés ou non, émanant des établissements d'enseignement et de recherche français ou étrangers, des laboratoires publics ou privés. 


\title{
Analytical expression of elastic rods at equilibrium under 3D strong anchoring boundary conditions
}

\author{
Olivier Ameline $^{\mathrm{a}, \mathrm{b}}$, Sinan Haliyo ${ }^{\mathrm{b}}$, Xingxi Huang ${ }^{\mathrm{a}, \mathrm{b}}$, Jean A. H. Cognet ${ }^{\mathrm{a}, *}$ \\ ${ }^{a}$ Sorbonne Université, UPMC University Paris 06, UMR 8237, LJP, BC 114, 4 Place Jussieu, F-75252 Paris Cedex 05, France \\ ${ }^{b}$ Sorbonne Université, UPMC University Paris 06, UMR 7222, ISIR, BC 173, 4 Place Jussieu, F-75252 Paris Cedex 05, France
}

\begin{abstract}
A general-purpose method is presented and implemented to express analytically one stationary configuration of an ideal 3D elastic rod when the end-to-end relative position and orientation are imposed. The mechanical equilibrium of such a rod is described by ordinary differential equations and parametrized by six scalar quantities. When one end of the rod is anchored, the analytical integration of these equations lead to one unique solution for given values of these six parameters. When the second end is also anchored, six additional nonlinear equations must be resolved to obtain parameter values that fit the targeted boundary conditions. We find one solution of these equations with a zero-finding algorithm, by taking initial guesses from a grid of potential candidates. We exhibit the symmetries of the problem, which reduces drastically the size of this grid and shortens the time of selection of an initial guess. The six variables used in the search algorithm, forces and moments at one end of the rod, are particularly adapted due to their unbounded definition domain. More than 850000 tests are performed in a large region of configurational space, and in $99.9 \%$ of cases the targeted boundary conditions are reached with short computation time and a precision better than $10^{-5}$. We propose extensions of the method to obtain many solutions instead of only one, using numerical continuation or starting from different initial guesses.
\end{abstract}

Keywords: boundary value problem, search algorithm, equilibrium of elastic rods

\section{Introduction}

Elastic rod models are used in different domains at different length-scales : supercoiled DNA modeling [1], animal locomotion [2, 3] or vibrissal system [4, 5] simulation, chirurgical intervention [6], nonlinear springs designing [7] and marine cables guiding 8 are examples. In some applications as resolving the conformation of single-stranded nucleic 5 acid structures [9 12, it is required to compute equilibrium configurations of elastic rods under specified geometric constraints. Such problems remain not entirely resolved and are the purpose of this paper.

The described work is done under the hypotheses of ideal elastica, where rods are taken inextensible, unshearable, isotropic, uniform and naturally straight and prismatic, with a hyperelastic material and linear constitutive relations. The Cosserat theory [13] is used to describe the configuration of these rods by the trajectory of their centre line together with a local reference frame, the Darboux frame, defined at each point of the trajectory and giving the orientation of cross-sections. Combining (i) the kinematic equations that describe the evolution of the Darboux frame along the trajectory, (ii) the equations of mechanical equilibrium and (iii) the constitutive relations between deformations and stresses, any rod configuration is characterized by 15 ordinary differential equations (ODEs).

Our global objective is to compute ideal 3D elastica configurations from imposed strong anchoring boundary conditions, i.e. relative position and orientation of the Darboux frame at both ends of the rod. Previous works [14 19 have demonstrated that the 15 ODEs of ideal elastica at equilibrium are integrable and have written closed form solutions as functions of a set of parameters. However, the influence of these parameters on the shape of the rod is complicated. As a consequence, finding the solution under strong anchoring boundary conditions can be achieved only by resolving a zero-finding problem, to obtain the parameters values that fit the targeted conditions. Many difficulties are encountered in such computations, due to the nonlinearity of the function to invert, as well as the complex constraints of its input space and the influence of initial guesses on convergence. Furthermore, several solutions of the problem may exist, but only one is usually obtained. To characterize the nonlinear behaviour that produces these different solutions, continuation techniques are particularly efficient 20-27. Yet, these methods compute multidimensional manifolds, thus continuous solution spaces to systems with more variables than equations. Therefore, providing pertinent initial guesses remains necessary to obtain the discrete set of solutions upon imposed strong anchoring boundary conditions. In [28, an effective general approach is proposed to deform an elastic rod from an initial state to a targeted configuration through control of anchoring boundary conditions. Still our problem is different, with initial state not specified

\footnotetext{
* Corresponding author

Email address: jean.cognet@upmc.fr (Jean A. H. Cognet)
} 
and final configuration unknown. Rod solutions to some specific boundary conditions have been implemented [29], but a general resolution is described in this article.

We provide a general method to express analytically one equilibrium configuration of ideal 3D elastica without self-contact that fulfill any arbitrary strong anchoring boundary conditions. Our approach is based on a complete formalisation of the equilibrium of rods, which has led to classifications of all shapes in a three-dimensional space [30]. In section 2, our objective is transcribed into an inverse problem. Six quantities called clamping parameters (three distances and three angles) are defined to characterize the boundary conditions. Then, two complementary

${ }_{35}$ parametrizations of ideal elastica shapes are introduced, leading to two analytical forward functions $q_{1}$ and $q_{2}$ with the clamping parameters as output. Function $q_{1}$ has six scalar inputs called physical parameters : they are dimensionless parameters from Landau et al. [31, which completely describe a 3D rod at equilibrium. These six parameters can be restricted to five thanks to a rotational invariance. However, the definition space of $q_{1}$ is bounded with non trivial frontiers. Function $q_{2}$ requires six scalar inputs called loading parameters (three coordinates of force and three coordinates of moment) without dimensional restriction, but its unbounded input space, $\mathbb{R}^{6}$, is particularly adapted for zero-finding algorithms. In section 3, the inverse problem is solved using Newton-Raphson's algorithm. Three definitions of the vector error are compared. To obtain a method that is at the same time efficient and robust, we found it best to take advantage of both functions, $q_{1}$ (physical parameters) and $q_{2}$ (loading parameters). The 5D function $q_{1}$ is used to provide initial guesses, and the $6 \mathrm{D}$ function $q_{2}$ is implemented in the search algorithm. One

45 symmetry of $q_{1}$ is used to increase the speed of the method. Results are exposed and discussed in section 4 . More than 850000 inversions are tested, that sample systematically a whole region of input space. Only forceless rods and planar Euler elasticae are not tested, because they are singular and would probably behave better in a specific implementation. Once these particular cases moved apart, the targeted boundary conditions are reached in $99.9 \%$ of cases with a precision better than $10^{-5}$. Extensions of the method are also proposed to compute many solutions instead of only one.

\section{Formulation of the problem}

\subsection{Equations of equilibrium of rods}

Ideal 3D elastic rods are described in a global reference frame $\left\{O, \mathbf{e}_{\mathrm{i}}, \mathbf{e}_{\mathrm{j}}, \mathbf{e}_{\mathrm{k}}\right\}$ by the trajectory of their centre line $\{\mathbf{r}(S)=\mathbf{O G}(S), S \in[0, L]\}$ and the local Darboux reference frame $\left\{G(S), \mathbf{d}_{i=1,2,3}(S), S \in[0, L]\right\}$ attached to cross-sections. The unit vector $\mathbf{d}_{3}(S)$ is taken as the local tangent $\mathbf{t}(S)$ to the trajectory.

Inextensibility and unshearability allow to write (denoting with an apostrophe the derivative with respect to $S$ )

$$
\mathbf{r}^{\prime}(S)=\mathbf{t}(S)=\mathbf{d}_{3}(S)
$$

The evolution of the Darboux frame along the trajectory is characterized by the Darboux vector $\boldsymbol{\Omega}$, through the relations

$$
\mathbf{d}_{i}^{\prime}(S)=\mathbf{\Omega}(S) \times \mathbf{d}_{i}(S)
$$

Noting $\mathbf{F}$ the force and $\mathbf{M}$ the moment acting across each section, the mechanical equilibrium is given by 31

$$
\left\{\begin{array}{l}
\mathbf{F}^{\prime}(S)=\mathbf{0} \\
\mathbf{M}^{\prime}(S)+\mathbf{t}(S) \times \mathbf{F}(S)=\mathbf{0} .
\end{array}\right.
$$

Hyperelasticity translates into constitutive relations between $\boldsymbol{\Omega}$ and $\mathbf{M}$, involving the bending rigidity $K_{0}$ and the torsion rigidity $K_{3}$ so that

$$
\mathbf{M}(S)=K_{0} \mathbf{\Omega}^{\perp \mathbf{d}_{3}}(S)+K_{3} \mathbf{\Omega}^{\| \mathbf{d}_{3}}(S),
$$

where $\boldsymbol{\Omega}^{\perp \mathbf{d}_{3}}(S)$ and $\boldsymbol{\Omega}^{\| \mathbf{d}_{3}}(S)$ are the components of $\boldsymbol{\Omega}(S)$ respectively perpendicular and parallel to $\mathbf{d}_{3}(S)$.

(1) to (4) lead to 15 ordinary differential equations that are sufficient to describe entirely any ideal $3 \mathrm{D}$ elastic rod shape at equilibrium. Considering that boundaries $\mathbf{r}(S=0)$ and $\mathbf{d}_{i}(S=0)$ are set by the choice of the global reference frame, the trajectory and the Darboux reference frame are functions of six parameters related directly or not to $\mathbf{F}$ and

6о $\mathbf{M}(S=0)$. As a result, expressing an elastica in a given problem consists in finding the corresponding values of these parameters. Generally, this cannot be done analytically and should be computed numerically through a root-finding algorithm.

\subsection{Clamping parameters}

In this article, we provide a method to express analytically the rod shapes of length $L$, bending rigidity $K_{0}$ and scaled torsional rigidity

$$
K_{30}=K_{3} / K_{0}
$$

that fulfill given strong anchoring boundary conditions, i.e. relative position and orientation of the Darboux reference frame between arc-length coordinates $S=0$ and $S=L$. 
Such boundary conditions can be represented by the following list of parameters (see Figure 1) :

$$
\boldsymbol{q}=\left[\eta_{1}, \eta_{2}, \eta_{3}, \psi_{R}, \theta_{R}, \varphi_{R}\right]^{T}
$$

the first three parameters being related to the relative position

$$
\Delta \mathbf{r}=\mathbf{r}(L)-\mathbf{r}(0)=\eta_{1} L \mathbf{d}_{1}(0)+\eta_{2} L \mathbf{d}_{2}(0)+\eta_{3} L \mathbf{d}_{3}(0)
$$

and the last three being the $Z Y Z$ Euler angles that orientate the Darboux basis at $S=L$ in the Darboux basis at $S=0$. These six parameters are called clamping parameters.

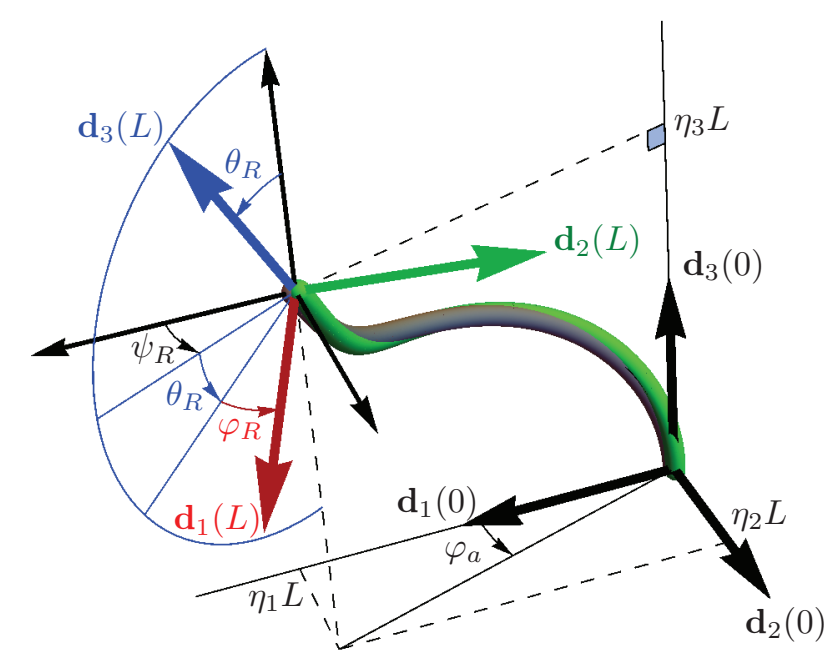

Figure 1: The six clamping parameters $\boldsymbol{q}$ that characterize strong anchoring boundary conditions, i.e. position and orientation of the Darboux frame at $S=L$ in the Darboux frame at $S=0:\left(\eta_{1}, \eta_{2}, \eta_{3}\right)$ are the coordinates of the position divided by $L$ and $\left(\psi_{R}, \theta_{R}, \varphi_{R}\right)$ are the $Z Y Z$ Euler angles. By convention, $\mathbf{d}_{2}$ of the Darboux reference frame is materialized on the surface of the rod with the light green color.

The method proposed here to impose these anchoring conditions uses two complementary parametrizations of elastic rods that satisfy equations (1) to (4). They are presented in the following two subsections.

\subsection{Physical parameters}

The first parametrization was proposed by L.D. Landau and E.M. Lifshitz [31. In their formalism, the arc-length coordinate, $s$, is defined with origin where $\mathbf{F}, \mathbf{d}_{3}$ and $\mathbf{M}$ are coplanar. Equations are written in a judicious reference frame $\mathfrak{L}=\left\{O, \mathbf{e}_{\mathbf{i}}, \mathbf{e}_{\mathbf{j}}, \mathbf{e}_{\mathrm{k}}\right\}$, positioned in relation to the point $s=0$ of the trajectory :

$$
\mathbf{e}_{\mathrm{k}}=\frac{1}{F} \mathbf{F}, \mathbf{e}_{\mathrm{i}}=-\mathbf{n}(0), \mathbf{e}_{\mathrm{j}}=\mathbf{e}_{\mathrm{k}} \times \mathbf{e}_{\mathrm{i}}, \mathbf{r}(0)=\frac{1}{F}\left(\mathbf{M}(0) \cdot \mathbf{e}_{\mathrm{j}}\right) \mathbf{e}_{\mathrm{i}}
$$

where $\mathbf{n}$ is the normal vector of Frenet and $F>0$ is the norm of the vector force $\mathbf{F}$. Case $F=0$ leads to helical rod trajectories and must be studied separately [19, 30]. Three dimensionless constants $\left(\lambda, t_{P}, a\right)$ are identified, such that $\forall s \in \mathbb{R}:$

$$
\begin{gathered}
\lambda=\frac{1}{\sqrt{K_{0} F}} \mathbf{M}(s) \cdot \mathbf{e}_{\mathrm{k}} \\
t_{P}=\frac{1}{\sqrt{K_{0} F}} \mathbf{M}(s) \cdot \mathbf{d}_{3}(s) \\
a=\frac{2}{F} \mathbf{F} \cdot \mathbf{d}_{3}(s)+\frac{1}{K_{0} F}\left\|\mathbf{M}^{\perp \mathbf{e}_{\mathrm{k}}}(s)\right\|^{2} .
\end{gathered}
$$

These constants are the three first integral of the problem, and parameter $a$ is directly related to the Hamiltonian 30. Then, the quantity $\mu=\sqrt{K_{0} / F}$ is used as unit of length and the ODEs are written using the cylindrical coordinates $(\rho, \phi, z)$ of axis $\left(O, \mathbf{e}_{\mathbf{k}}\right)$. The resulting expressions are integrated analytically [16, 19, 30] from $-\infty$ to $+\infty$, leading to a full geometric and mechanical description of infinite rods $: \forall s \in \mathbb{R}$,

$$
\left\{\begin{array}{l}
\mathbf{r}(s)=\mu \overline{\mathbf{r}}\left(s, \lambda, t_{P}, a\right) \\
(\psi, \theta, \varphi)(s)=(\psi, \theta, \bar{\varphi})\left(s, \lambda, t_{P}, a, K_{30}\right)+\left(0,0, \varphi_{0}\right) \\
(\mathbf{F}, \mathbf{M})(s)=K_{0} / \mu^{2}\left(\mathbf{e}_{\mathrm{k}}, \mathbf{e}_{\mathrm{k}} \times \mathbf{r}(s)+\mu \lambda \mathbf{e}_{\mathrm{k}}\right)
\end{array}\right.
$$


where $(\psi, \theta, \varphi)$ are the $Z Y Z$ Euler angles of the Darboux reference frame. Equations of geometry $12 \mathrm{a})$ and $(12 \mathrm{~b})$ have closed-form expressions involving Jacobi elliptic functions and integrals. Parameters $\left(\lambda, t_{P}, a\right)$ are sufficient to describe the infinite dimensionless trajectory $\overline{\mathbf{r}}(s)$, and the scaling factor $\mu$ applies an homothetic transformation to set the geometry at the correct size. The radial coordinate $\rho(s)$ is even, periodic with period $s_{\text {per }}$ and strictly decreasing in $\left[0, s_{\text {per }} / 2\right]$. Its dimensionless period $s_{p e r} / \mu$ is known analytically as a function of $\left(\lambda, t_{P}, a\right)$. The value $\varphi_{0}=\varphi(s=0)$ is required as a reference for the intrinsic rotation $\varphi$ of the Darboux frame, as well as the scaled torsional rigidity $K_{30}$ to describe the function $\varphi(s)$.

Finally, two additional parameters $\left(s_{i n i} / \mu, L / \mu\right)$ are necessary to select a part of infinite dimensionless trajectory, where $s_{i n i} / \mu$ is the starting arc-length coordinate of the finite rod and $L / \mu$ is its nondimensionalized length. With this setting, the arc-length coordinate $s \in \mathbb{R}$ of the infinite trajectory is related to the arc-length coordinate $S \in[0, L]$ of the finite trajectory by

$$
s=s_{i n i}+S
$$

As a result, the six quantities

$$
\boldsymbol{p}=\left[\lambda, t_{P}, a, \varphi_{0}, s_{i n i} / \mu, L / \mu\right]^{T}
$$

are sufficient to express analytically all finite dimensionless rod shapes of a given scaled torsional rigidity $K_{30}$. The value of $L$ is then required to obtain the dimensioned shape, along with the value of $K_{0}$ to describe the mechanical state. The six quantities $\boldsymbol{p}$ are called physical parameters.

The definition domain of all these parameters is not trivial. As $\lambda$ and $t_{P}$ are proportional to components of the moment, they are defined in all $\mathbb{R}$. But it has been demonstrated in 30 that $a$ is bounded below by a surface $a_{M i n}\left(\lambda, t_{P}\right)$, plotted in Figure 2. Then, angle $\varphi_{0}$ varies in all $[0,2 \pi]$. Due to the periodicity, $s_{\text {ini }} / \mu$ is limited to $\left[0, s_{\text {per }} / \mu\right]$. Obviously, $L / \mu$ is positive.

Although the definition domain is difficult, this parametrization has significant advantages because it obeys to a hierarchy that leads to mathematical simplifications. In particular, the dimension of space can be reduced from six to five by noting with equation (12) that parameter $\varphi_{0}$ yields only a shift in intrinsic rotation $\varphi$. When the rod shape is expressed in the Darboux reference frame at $S=0$, this shift is accompanied by a rotation of the rod trajectory around its tangent at $S=0$. Thus, the transformation

$$
\left[\lambda, t_{P}, a, \varphi_{0}, \frac{s_{i n i}}{\mu}, \frac{L}{\mu}\right]^{T} \longmapsto\left[\lambda, t_{P}, a, \varphi_{0}-\varphi_{a}, \frac{s_{i n i}}{\mu}, \frac{L}{\mu}\right]^{T}
$$

implies

$$
\left[\begin{array}{c}
\eta_{1} \\
\eta_{2} \\
\eta_{3} \\
\psi_{R} \\
\theta_{R} \\
\varphi_{R}
\end{array}\right] \longmapsto\left[\begin{array}{c}
\cos \left(\varphi_{a}\right) \eta_{1}-\sin \left(\varphi_{a}\right) \eta_{2} \\
\sin \left(\varphi_{a}\right) \eta_{1}+\cos \left(\varphi_{a}\right) \eta_{2} \\
\eta_{3} \\
\psi_{R}+\varphi_{a} \\
\theta_{R} \\
\varphi_{R}-\varphi_{a}
\end{array}\right]
$$

As a consequence, the study can be limited to the rod shapes for which $\eta_{1} \geq 0$ and $\eta_{2}=0$. Only five clamping parameters are then sufficient to characterize the boundary conditions,

$$
\boldsymbol{q}_{5 D}=\left[\eta_{1}, \eta_{3}, \psi_{R}, \theta_{R}, \varphi_{R}\right]^{T} .
$$

In the same way, the five physical parameters

$$
\boldsymbol{p}_{5 D}=\left[\lambda, t_{P}, a, s_{i n i} / \mu, L / \mu\right]^{T}
$$

are sufficient to express the geometry : the value of $\varphi_{0}$ that yields $\eta_{1} \geq 0$ and $\eta_{2}=0$ is obtained readily using transformations (15) and (16). Finally, every elastic rod shape for which $\eta_{1}<0$ or $\eta_{2} \neq 0$ can be related bijectively to a rod shape $\eta_{1} \geq 0$ and $\eta_{2}=0$ through transformations $(15)$ and $(16)$, taking $\varphi_{a}$ as (see Figure 1 )

$$
\varphi_{a}=\left\{\begin{array}{ll}
\operatorname{sign}\left(\eta_{2}\right) \pi / 2 & \text { if } \eta_{1}=0 \\
\arctan \left(\eta_{2} / \eta_{1}\right) & \text { if } \eta_{1}>0 \\
\pi+\arctan \left(\eta_{2} / \eta_{1}\right) & \text { otherwise }
\end{array} .\right.
$$

It results that six targeted clamping parameters $\boldsymbol{q}$ can be imposed by inverting the 5D function (known analytically)

$$
\begin{aligned}
q_{1}: \mathfrak{D} \subset \mathbb{R}^{5} & \rightarrow \mathbb{R}^{5} \\
\boldsymbol{p}_{5 D} & \mapsto \boldsymbol{q}_{5 D} .
\end{aligned}
$$

This dimensional reduction is possible because the classical Landau and Lifshitz formalism gathers all conformations with the same trajectory but with different Darboux frames. 


\subsection{Loading parameters}

The second parametrization of rod shapes of length $L$, bending rigidity $K_{0}$ and scaled torsional rigidity $K_{30}$ is proposed with the components of the force and moment in the initial Darboux reference frame, $F_{i 0}=\mathbf{F} \cdot \mathbf{d}_{i}(S=0)$ and $M_{i 0}=\mathbf{M}(S=0) \cdot \mathbf{d}_{i}(S=0)$ :

$$
\boldsymbol{f}=\left[F_{10}, F_{20}, F_{30}, M_{10}, M_{20}, M_{30}\right]^{T} .
$$

The six quantities in $\boldsymbol{f}$, called loading parameters, are defined in all $\mathbb{R}^{6}$. Thus, the function

$$
\begin{aligned}
q_{2}: \mathbb{R}^{6} & \rightarrow \mathbb{R}^{6} \\
\boldsymbol{f} & \mapsto \boldsymbol{q}
\end{aligned}
$$

is particularly robust for implementation in root-finding algorithms. Yet, it cannot be restricted to five dimensions as $q_{1}$.

This function is known analytically, because it is possible to build bijective relations between the loading parameters $\boldsymbol{f}$ and the physical parameters $\boldsymbol{p}$ defined in subsection 2.3 . Consider a rod of length $L$, bending rigidity $K_{0}$ and scaled torsional rigidity $K_{30}$. Obviously, $\boldsymbol{f}$ is deduced from $\boldsymbol{p}$ as the physical parameters provide an entire geometric and mechanical description of the elastic rod through equations $(12)$. Conversely, suppose that $\boldsymbol{f}$ is known and $\boldsymbol{p}$ is searched. Then the unit of length $\mu$, used in 2.3 , is known as it is a function of $K_{0}$ and $F$. As a consequence, the dimensionless length $L / \mu$ is obtained readily, and parameters $\left(\lambda, t_{P}, a\right)$ are computed using equations (8) to (11). Then, from the component of $\left(12 \mathrm{c}\right.$ ) orthogonal to $\mathbf{e}_{\mathrm{k}}$ and its derivative with respect to $s$, it can be noticed that

$$
\begin{gathered}
\rho_{i n i}=\rho\left(s_{i n i}\right)=\left\|\mathbf{M}(S=0)^{\perp \mathbf{e}_{\mathrm{k}}}\right\| / F \\
\rho_{\text {ini }}^{\prime}=\rho^{\prime}\left(s_{i n i}\right)=\frac{1}{\rho_{\text {ini }} F^{2}} \mathbf{d}_{3}(S=0) \times \mathbf{M}(S=0) \cdot \mathbf{F} .
\end{gathered}
$$

As defined above, $\rho(s)$ is the radial coordinate of the trajectory and $\rho^{\prime}(s)$ is its derivative with respect to $s$. Thus, $s_{i n i}$ is obtained by inverting equation $12 \mathrm{a}$. As the function $\rho(s)$ is even, periodic with period $s_{\text {per }}$ and strictly decreasing in $\left[0, s_{\text {per }} / 2\right]$ it follows that

$$
s_{i n i} \equiv-\operatorname{sign}\left(\rho_{i n i}^{\prime}\right) \rho^{-1}\left(\rho_{i n i}\right) \quad\left[s_{p e r}\right],
$$

where $\rho^{-1}\left(\rho_{\text {ini }}\right)$ is the unique preimage of $\rho_{i n i}$ in $\left[0, s_{\text {per }} / 2\right]$ under the function $\rho(s)$. This value is straightforward to obtain as $\rho(s)$ only involves the sinus function of Jacobi, whose inverse is defined analytically. Finally, to obtain the initial intrinsic rotation $\varphi_{0}$, the directors $\mathbf{d}_{i}\left(s=s_{\text {ini }}\right)$ are written in Landau's reference frame $\mathfrak{L}=\left\{O, \mathbf{e}_{\mathrm{i}}, \mathbf{e}_{\mathrm{j}}, \mathbf{e}_{\mathrm{k}}\right\}$ defined by equations (8). This is trivial for $\mathbf{d}_{3}$ because parameters $\left\{\lambda, t_{P}, a, \mu\right\}$ has been obtained, which provides the expression of the trajectory in $\mathfrak{L}$. For $\mathbf{d}_{1}, \mathbf{F}$ and $\mathbf{M}$ are expressed in $\mathfrak{L}$ using equation $(12 \mathrm{c})$, and their expression in the Darboux frame at $s=s_{i n i}$ (equivalently $S=0$ ) imply

$$
\begin{aligned}
\mathbf{d}_{\mathbf{1}}\left(s_{i n i}\right) & =\frac{F_{10} \mathbf{F}^{\perp \mathbf{d}_{3}}-F_{20} \mathbf{d}_{3} \times \mathbf{F}^{\perp \mathbf{d}_{3}}}{F_{10}^{2}+F_{20}^{2}}\left(s_{i n i}\right) \\
& =\frac{M_{10} \mathbf{M}^{\perp \mathbf{d}_{3}}-M_{20} \mathbf{d}_{3} \times \mathbf{M}^{\perp \mathbf{d}_{3}}}{M_{10}^{2}+M_{20}^{2}}\left(s_{i n i}\right) .
\end{aligned}
$$

Then $\mathbf{d}_{2}=\mathbf{d}_{3} \times \mathbf{d}_{1}$. With $\mathbf{d}_{i}\left(s_{\text {ini }}\right)$ written in $\mathfrak{L}$, the intrinsic rotation $\varphi\left(s_{\text {ini }}\right)$ is obtained and equation $12 \mathrm{~b}$ yields

$$
\varphi_{0}=\varphi\left(s_{i n i}\right)-\bar{\varphi}\left(s_{i n i}, \lambda, t_{P}, a, K_{30}\right) .
$$

Although analytic expressions are faster, it is sometimes more interesting to integrate equations (1) to (4) numerically to avoid singularities. In this case, the geometry can be expressed directly in $\left\{G, \mathbf{d}_{i=1,2,3}\right\}(S=0)$ with the loading parameters $\boldsymbol{f}$. It is then straightforward to obtain the clamping parameters $\boldsymbol{q}$. Such numerical integration is better for elastic rods $t_{P}= \pm \lambda$, where singularities are observed in the analytic equations. This is why it is automatically chosen when $\left|t_{P} \pm \lambda\right|<10^{-2}$, using the Bulirsch-Stoer method with Richardson's extrapolation [32, 33].

\section{Resolution of the inverse problem}

The problem exposed in 2.2 , i.e. imposing strong anchoring conditions to elastic rods, has been translated in 2.3 and 2.4 into the inversion of function $q_{1}$ or $q_{2}$. To compute a rod of clamping parameters $\boldsymbol{q}$, one has to find values of the five physical parameters $\boldsymbol{p}_{5 D}$ such that $q_{1}\left(\boldsymbol{p}_{5 D}\right)=\boldsymbol{q}_{5 D}$ or of the six loading parameters $\boldsymbol{f}$ such that $q_{2}(\boldsymbol{f})=\boldsymbol{q}$. Before going further, note that this problem only depends on $K_{30}=K_{3} / K_{0}$ and not on the values of $L$ and $K_{0}$.

deed, the clamping parameters have been defined in (6) with distances divided by $L$, so that any change of rod length can be obtained by an homothetic transformation. Regarding the bending rigidity $K_{0}$, it has no influence on the geometry but only on the mechanical state, because the imposed conditions $\boldsymbol{q}$ are geometric constraints. In this article, the value of $K_{30}$ is taken at $K_{30}=1$. 
The proposed method of inversion makes use of Newton-Raphson's algorithm. Three definitions of the error, described in subsection 3.1, are compared. Then, the key idea is to use the 5D analytic function $q_{1}$ to provide initial guesses to the algorithm (subsection 3.2) and the $6 \mathrm{D}$ analytic/numeric function $q_{2}$ to invert (subsection 3.3). Tests and results are exposed and discussed in section 4 .

\subsection{Error definitions}

In an iterative process, proper definition of the error function $\boldsymbol{\epsilon}$ to minimize is crucial. Noting $\overline{\boldsymbol{q}}$ the current clamping parameters and $\boldsymbol{q}$ the target, it should verify

$$
\epsilon(\bar{q})=0 \Longleftrightarrow \bar{q}=q
$$

A first proposition for $\boldsymbol{\epsilon}(\overline{\boldsymbol{q}})$ would be

$$
\epsilon_{1}(\bar{q})=q-\bar{q}
$$

This definition is simple and fast, but the discontinuities of Euler angles, defined in finite intervals, may cause divergences of the method. Other choices of angular error are possible by comparing in the same reference frame the unit vectors $\overline{\mathbf{d}}_{i}$ and $\mathbf{d}_{i}$ oriented by the Euler angles in $\overline{\boldsymbol{q}}$ and $\boldsymbol{q}$ respectively. In particular, projected angles are defined as follows :

$$
\boldsymbol{\epsilon}_{\mathbf{2}}(\overline{\boldsymbol{q}})=\left[\eta_{1}-\bar{\eta}_{1}, \eta_{2}-\bar{\eta}_{2}, \eta_{3}-\bar{\eta}_{3}, \theta_{1}, \theta_{2}, \theta_{3}\right]^{T}
$$

where $\left(\theta_{1}, \theta_{2}, \theta_{3}\right)$ are signed angles such that

$$
\theta_{1}=\left(\overline{\mathbf{d}}_{2}, \mathbf{d}_{2}^{\perp \overline{\mathbf{d}}_{1}}\right), \theta_{2}=\left(\overline{\mathbf{d}}_{3}, \mathbf{d}_{3}^{\perp \overline{\mathbf{d}}_{2}}\right), \theta_{3}=\left(\overline{\mathbf{d}}_{1}, \mathbf{d}_{1}^{\perp \overline{\mathbf{d}}_{3}}\right) .
$$

If Newton-Raphson's algorithm initiates from rod shapes close to the solution, these angles are small and discontinuities can be avoided by defining them in $[-\pi, \pi[$. Finally, a third definition is proposed by considering the rotation about unit vector $\mathbf{u}$ and of angle $\gamma \in\left[0, \pi\left[\right.\right.$ that transforms basis $\left\{\overline{\mathbf{d}}_{i}\right\}$ into $\left\{\mathbf{d}_{i}\right\}$ :

$$
\boldsymbol{\epsilon}_{\mathbf{3}}(\overline{\boldsymbol{q}})=\left[\eta_{1}-\bar{\eta}_{1}, \eta_{2}-\bar{\eta}_{2}, \eta_{3}-\bar{\eta}_{3}, v_{1}, v_{2}, v_{3}\right]^{T},
$$

where $\mathbf{v}=v_{1} \overline{\mathbf{d}}_{1}+v_{2} \overline{\mathbf{d}}_{2}+v_{3} \overline{\mathbf{d}}_{3}=\sin (\gamma / 2) \mathbf{u}$.

These three error functions can also be defined for $5 \mathrm{D}$ clamping parameters $\overline{\boldsymbol{q}}_{5 D}$ and $\boldsymbol{q}_{5 D}$.

\subsection{Initial guesses}

One difficulty with Newton-Raphson's algorithm is that the user must provide initial values of physical parameters $\boldsymbol{p}$ sufficiently close to the solution. To address this issue, a grid $\mathcal{G}$ of initial guesses is built using the function $q_{1}$ rather than $q_{2}$, because it is five-dimensional and because it can be restricted further by using symmetries and classifications presented in [30. A subset $\mathfrak{G}$ of the definition domain $\mathfrak{D}$ is discretized, and for each point of the resulting discrete space $\mathfrak{G}_{d} \subset \mathfrak{G}$, the corresponding image under $q_{1}$ is computed :

$$
\mathcal{G}=\left\{\left(\boldsymbol{p}_{5 D}, q_{1}\left(\boldsymbol{p}_{5 D}\right)\right), \boldsymbol{p}_{5 D} \in \mathfrak{G}_{d}\right\}
$$

The grid $\mathcal{G}$ is computed only once, then stored and used for any boundary conditions by choosing as initial guess the element $\boldsymbol{p}_{5 D}$ of $\mathfrak{G}_{d}$ whose image is the closest to the targeted clamping parameters $\boldsymbol{q}_{5 D}$. This method is simple, but it is time-consuming as it requires to scan entirely the grid $\mathcal{G}$. Furthermore, only rod solutions in the subset $\mathfrak{G}$ have a significant chance to be obtained, and the convergence will be improved by reducing the step of discretization. Thus, a compromise between high resolution, high boundaries of $\mathfrak{G}$ and low number of elements in $\mathcal{G}$ must be found.

This is a strong justification to use the $5 \mathrm{D}$ function $q_{1}$ rather than the $6 \mathrm{D}$ function $q_{2}$ in order to reduce drastically the total size of the grid. Then, to obtain initial guesses faster, the distance is evaluated through the norm of the fastest error function $\boldsymbol{\epsilon}_{\mathbf{1}}$, even if $\boldsymbol{\epsilon}_{\mathbf{2}}$ or $\boldsymbol{\epsilon}_{\mathbf{3}}$ is used in the inversion algorithm. Finally, it is possible to reduce the number of points of the grid $\mathcal{G}$ by using the symmetries of $q_{1}$. Due to the properties of elliptic functions, it can be demonstrated that the transformation

$$
\left[\lambda, t_{P}, a, \frac{s_{i n i}}{\mu}, \frac{L}{\mu}\right]^{T} \longmapsto\left[-\lambda,-t_{P}, a, \frac{s_{i n i}}{\mu}, \frac{L}{\mu}\right]^{T}
$$

implies

$$
\left[\eta_{1}, \eta_{3}, \psi_{R}, \theta_{R}, \varphi_{R}\right]^{T} \longmapsto\left[\eta_{1}, \eta_{3}, 2 \pi-\psi_{R}, \theta_{R}, 2 \pi-\varphi_{R}\right]^{T}
$$

With this property, it is possible to construct grids $\mathcal{G}$ whose elements $q_{1}\left(\boldsymbol{p}_{5 D}\right)$ are such that $\varphi_{R} \leq \pi$. For targeted clamping parameters with $\varphi_{R}>\pi$, the inverse of transformation 35 must be applied before using Newton-Raphson's algorithm with such grids. When the algorithm is completed, transformation (34) is applied to obtain the solution. This space reduction lead to smaller grids without changing the resolution or the boundaries. 


\subsection{Inversion}

Once given an initial set of physical parameters, the corresponding values of loading parameters $\boldsymbol{f}_{0}$ are calculated and used to start Newton-Raphson's algorithm [33] (see algorithms 1 and 22). The Jacobian matrix $\boldsymbol{J}$ is computed numerically at each incrementation of variable $\boldsymbol{f}_{s}$ using a second-order central finite difference. As the convergence is highly dependent on the accuracy of this finite-difference scheme, the step $h$ must be chosen carefully. Thus, the value $h=10^{-5}\left\|\boldsymbol{f}_{s}\right\|$ is taken by default and if the algorithm diverges, it is restarted using Ridders' iterative method [34 to calculate the derivatives. The Jacobian matrix is used to compute the Newton step $\boldsymbol{d}=\delta \boldsymbol{f}_{s}$ such that the approximate error at order 1 vanishes at $\boldsymbol{f}_{s}+\boldsymbol{d}$. To ensure the convergence of the algorithm, a line search is performed using Armijo's rule [35]. It consists of imposing a condition of linear decreasing at each step (see algorithm 2, line 7). If this condition is not satisfied by the Newton step, a suitable step is taken by backtracking gradually along the descent direction $\boldsymbol{d} /\|\boldsymbol{d}\|$. Yet for critical points where the determinant of $\boldsymbol{J}$ is null or very close to zero, it may occur that no suitable step is obtained. As function $q_{2}$ is nonlinear and non-convex and because the dimension is high, such points may occur frequently [36, 37. When they are met, Newton-Raphson's algorithm is stopped (algorithm 1 , line 12 and restarted after modifying the initial guess with the function

$$
\left[\lambda, t_{P}, a, \frac{s_{i n i}}{\mu}, \frac{L}{\mu}\right]^{T} \longmapsto\left[\lambda, t_{P}, a, \frac{s_{i n i}}{\mu}+\frac{n_{\text {ini }}}{10} \frac{s_{p e r}}{\mu}, \frac{L}{\mu}\right]^{T}
$$

At most nine other points are tested to initiate the algorithm, with $n_{\text {ini }} \in\{1, \ldots, 9\}$. These initial guesses are relatively close to the first one, which is close to the solution, and we observed that modifying parameter $s_{\text {ini }}$ often suffices to avoid the singularity.

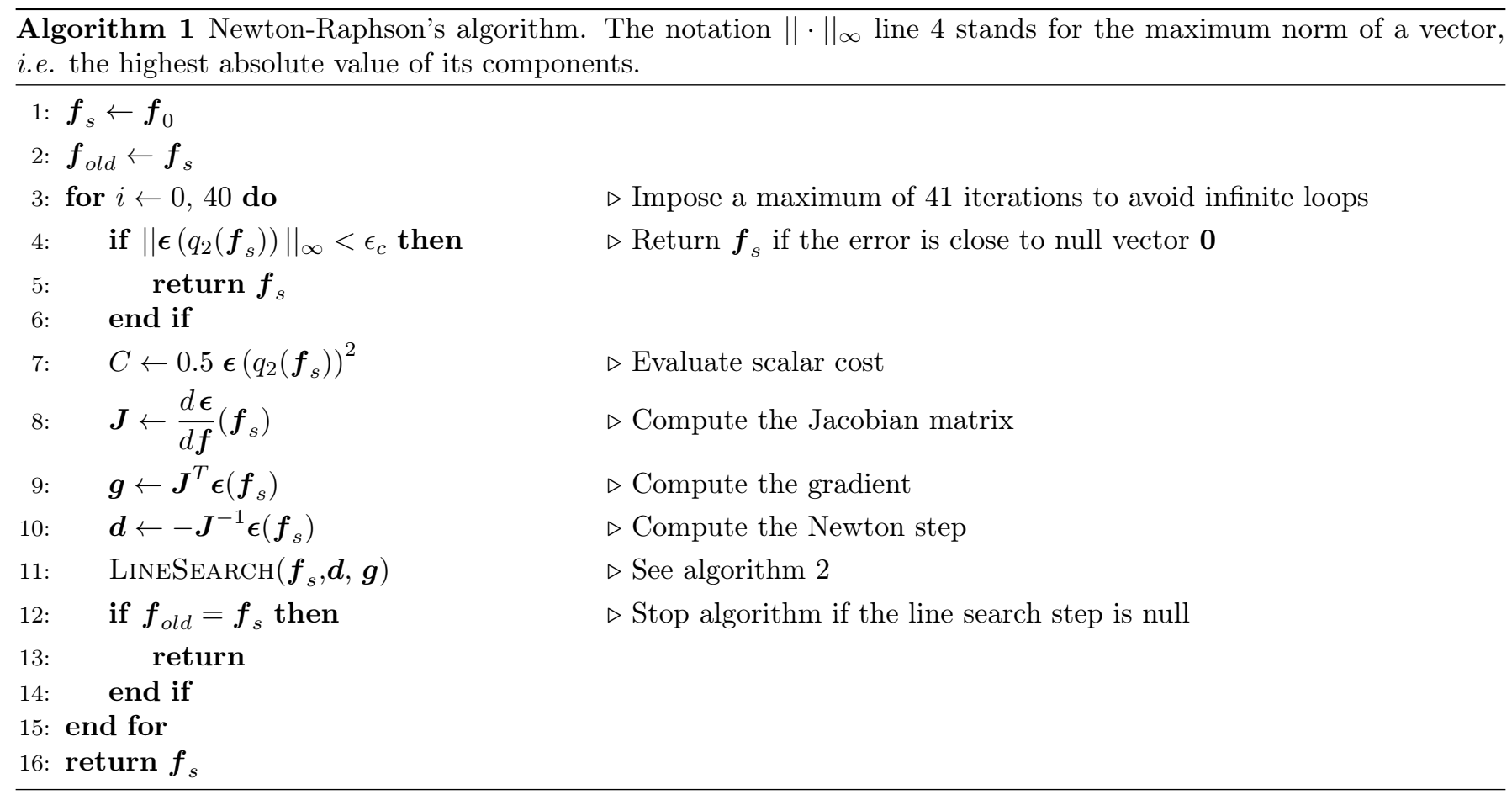

\section{Results and discussion}

\subsection{Indicators of performance}

Finally, the method is tested on a large number of inversions. As explained in subsection 3.2 a grid $\mathcal{G}$ must be constructed to provide initial guesses. It follows that only rod solutions in a subset $\mathfrak{G}$ of the definition domain of $q_{1}$ are expected to be obtained. As a consequence, the image of $\mathfrak{G}$ under $q_{1}$ should be known to ensure that there exists rods in $\mathfrak{G}$ that fit the targeted anchoring conditions. As this image may be difficult to characterize, one procedure to test exclusively the efficiency of the inversion - and not the existence of a solution - is to discretise a test subset $\mathfrak{H} \subset \mathfrak{G}$ and for each point $\boldsymbol{p}_{5 D}$ of the resulting discrete space $\mathfrak{H}_{d} \subset \mathfrak{H}$, apply the following testing path :

$$
\boldsymbol{p}_{5 D} \stackrel{q_{1}}{\longmapsto} \boldsymbol{q}_{5 D} \stackrel{q_{1}^{-1}}{\longmapsto} \overline{\boldsymbol{p}}_{5 D} \stackrel{q_{1}}{\longmapsto} \overline{\boldsymbol{q}}_{5 D} .
$$




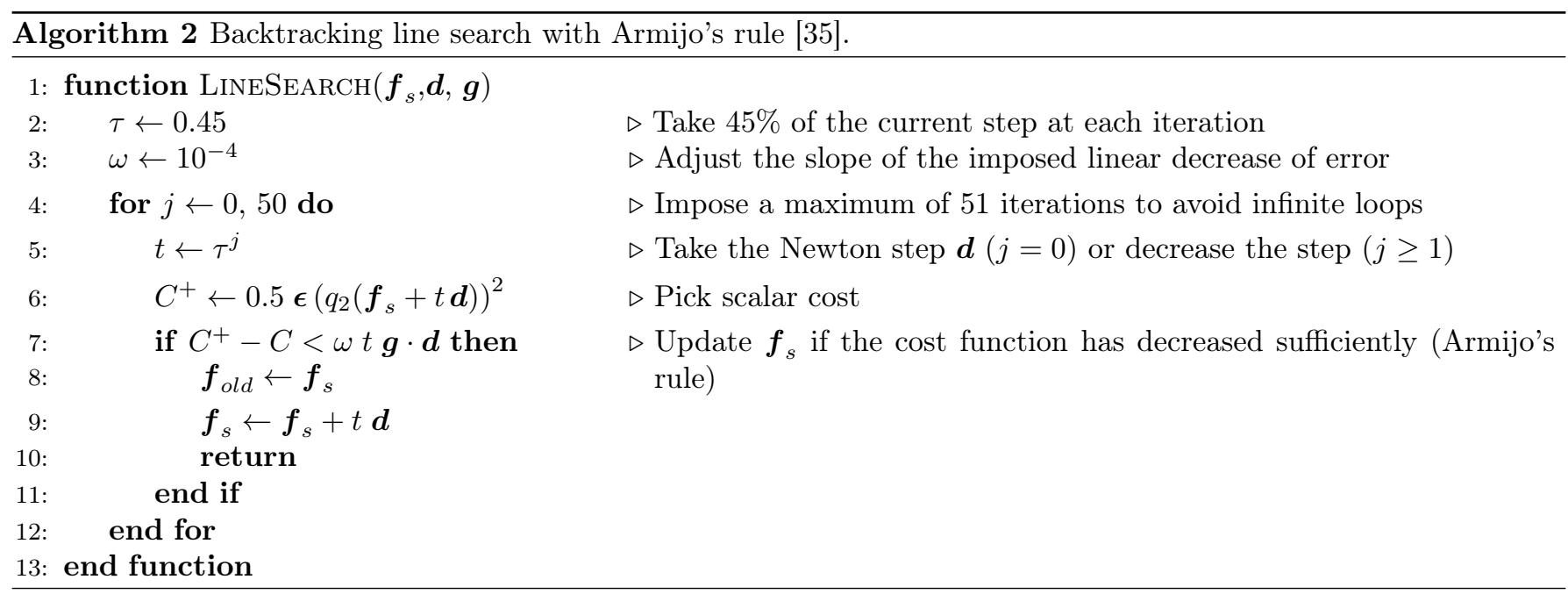

Mapping $q_{1}$ to obtain $\boldsymbol{q}_{5 D}$ and $\overline{\boldsymbol{q}}_{5 D}$ is always carried out with analytical expressions. This generates the set of inversions

$$
\mathcal{L}=\left\{\left(\boldsymbol{p}_{5 D}, \boldsymbol{q}_{5 D}, \overline{\boldsymbol{p}}_{5 D}, \overline{\boldsymbol{q}}_{5 D}\right), \boldsymbol{p}_{5 D} \in \mathfrak{H}_{d}\right\} .
$$

From this set, three indicators of performance are defined. First, the rate of success at $10^{-5}$ indicates the percentage of points in $\mathcal{L}$ that satisfy

$$
\left\|\boldsymbol{q}_{5 D}-\overline{\boldsymbol{q}}_{5 D}\right\|_{\infty}<10^{-5}
$$

where $\|\cdot\|_{\infty}$ is the maximum norm, i.e. the highest absolute value of the components. Second, the average time taken by one inversion is the total time of set $\mathcal{L}$ generation divided by the number of elements. Third, the average number of iterations gives the convergence speed of the inversion algorithm. The computations are performed on a Intel i7-5930K, 3.5GHz.

\subsection{Initialization grid}

Figure 2 shows the region of space $\mathfrak{G}$ in which the initialization grid is generated, as projected on space $\left\{\lambda, t_{P}, a\right\}$. This region is characterized by its size $l \in \mathbb{N}$ :

$$
\boldsymbol{p}_{5 D} \in \mathfrak{G} \Longleftrightarrow\left\{\begin{array}{l}
\left(\lambda, t_{P}\right) \in[-l, l]^{2} \\
a \in\left[a_{M i n}\left(\lambda, t_{P}\right), l^{2}\right] \\
s_{\text {ini }} / \mu \in\left[0,0.95 s_{\text {per }} / \mu\right] \\
L / \mu \in\left[0.1, s_{\text {per }} / \mu\right]
\end{array} .\right.
$$

The upper bound $l^{2}$ for $a$ has been chosen to be always greater than the lower bound $a_{M i n}\left(\lambda, t_{P}\right)$. The dimensionless length $L / \mu$ is limited to one period. Considering the general geometric structure of ideal elastica's equilibrium configurations [30, it appears sufficient for many applications, but solutions with larger $L / \mu$ may then be obtained by numerical continuation. Space $\mathfrak{G}$ is discretized using the same constant step $\delta$ for each physical parameter $\boldsymbol{p}_{k}$, always starting from the lower bound as exposed in Figure 2. Yet if symmetry rule (35) is used, parameter $t_{P}$ is taken from 0 to $l$ and transformation (34) is applied to ensure $\varphi_{R} \leq \pi$. Furthermore, we noticed during the implementation that all points $t_{P}=0$ are singular, i.e. yield null determinant of the Jacobian matrix. Thus, these points are replaced in the initialization grid by points $t_{P}=10^{-3}$, without changing the other physical parameters.

\subsection{Tested points}

The set $\mathcal{L}$ of inversions is constructed carefully to ensure that tested points are different from initial guesses. In this objective, the bounds of the covered space $\mathfrak{H} \subset \mathfrak{G}$ are positioned at a distance $\delta / 2$ from those of $\mathfrak{G}$ (see Figure 2). Then, the same constant step of discretization $\alpha \delta$ is used for all physical parameters. This implies that the number of tested inversions cannot be imposed, but it can be regulated with the value of $\alpha$. This value is chosen such that the number of intervals for parameters $\lambda$ and $t_{P}$ is exactly $2 l+1$, thus

$$
\alpha_{1}=\frac{2 l-\delta}{(2 l+1) \delta}
$$

Yet, when the computational time is sufficiently short, a smaller value $\alpha_{2}=\alpha_{1} / 2$ is used to test more inversions. To avoid trivial trajectories, only rod shapes that satisfy

$$
\|\Delta \mathbf{r}\| / L<0.95
$$




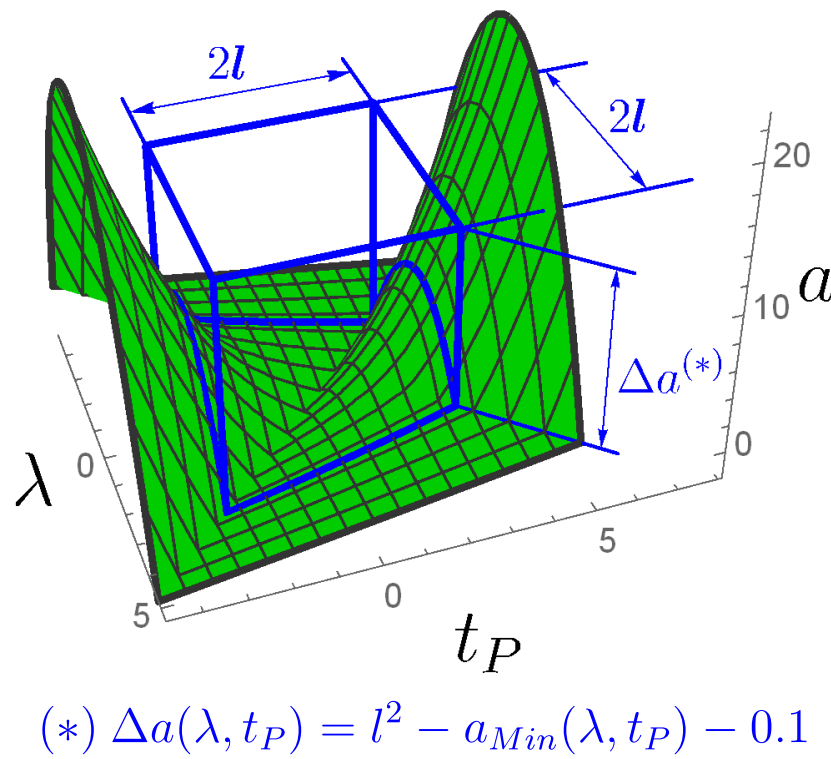

(a)

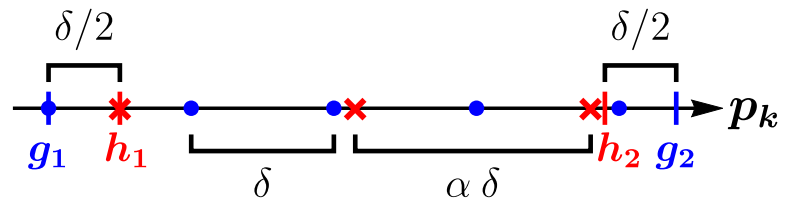

(b)

Figure 2: Description of the initial guesses $\boldsymbol{p}_{5 D} \in \mathfrak{G}_{d} \subset \mathfrak{G}$ used to compute the grid $\mathcal{G}$ through equation $(33)$ and of the corresponding tested points $\boldsymbol{p}_{5 D} \in \mathfrak{H}_{d} \subset \mathfrak{H}$ from which path 37 is applied and set $\mathcal{L}$ is generated. (a) Plot of the lower bound surface $a_{M} i_{n}\left(\lambda, t_{P}\right)$ that delimits the definition domain of $q_{1}$ together with the boundaries of $\mathfrak{G}$ characterized by size $l \in \mathbb{N}$. $(b)$ The discretization of $\mathfrak{G}$ and $\mathfrak{H}$ is achieved in each dimension within the bounds $\left(g_{1}, g_{2}\right)$ of $\mathfrak{G}$ and $\left(h_{1}, h_{2}\right)$ of $\mathfrak{H}$ respectively; $\boldsymbol{p}_{k}(k=1 \ldots 5)$ stands for $a, \lambda, t_{P}, s_{i n i} / \mu$ or $L / \mu$. Dots represent the initial guesses, evenly spaced from the lower bound $g_{1}$ with the same step $\delta$ in each dimension. Crosses represent the tested points, generated similarly from $h_{1}$ with the step $\alpha \delta$.

are maintained in the sets of inversions. Rods $\lambda=t_{P}=0$, which correspond to planar Euler elastica, are not tested because they are singular: this special case must be implemented separately.

\subsection{Assessments} definitions of subsection 3.1. Four initialization grids are tested, with the same size $l=2$ and different values of $\delta$. The corresponding sets of inversions are generated with each error $\boldsymbol{\epsilon}_{\mathbf{1}}, \boldsymbol{\epsilon}_{\mathbf{2}}$ and $\boldsymbol{\epsilon}_{\mathbf{3}}$. Results are shown in Figure 3

As expected, decreasing $\delta$ improves the rate of successful inversions and reduces the average number of iterations. Then, optimal time per inversion is obtained for intermediary values of discretization step. When $\delta$ is too large, for which the Jacobian is null or close to zero). Each singular point requires time costly high precision Ridders's algorithm to compute the Jacobian matrix, as well as additional tests with other initial guesses to try to get around the singularity. On the other hand, too small values of $\delta$ entail large grids $\mathcal{G}$ thus heavy computation cost to provide initial guesses. The value $\delta=0.5$ seems to satisfy both requirements of high success rate and speed. Taking $\delta=0.5$, the definition of the error, despite the discontinuities and singularities attached to Euler angles. When $\delta$ is large, though, significantly better results are obtained with $\boldsymbol{\epsilon}_{\mathbf{1}}$ (see for instance $\delta=2$ in Figure 3). A first reason is that the composition of functions in $\boldsymbol{\epsilon}_{\mathbf{2}}$ and $\boldsymbol{\epsilon}_{\mathbf{3}}$ lead to more frequent singularities. Another reason is that $\boldsymbol{\epsilon}_{\mathbf{1}}$ is used to scan the grid $\mathcal{G}$ and provide initial guesses, even if $\boldsymbol{\epsilon}_{2}$ or $\boldsymbol{\epsilon}_{3}$ are used in search algorithm. This saves time for large grids $\mathcal{G}$, but lowers too much the quality of initial guesses for small grids. To illustrate this, one additional test (not in Figure 3) has been performed with $l=2$ and $\delta=2$ using $\boldsymbol{\epsilon}_{\mathbf{3}}$, both to scan the grid and run the root-finding algorithm : the rate of success increased from $94 \%$ to $94.9 \%$ and the time per inversion decreased from 263 ms to 171 ms. Finally, considering success rate, speed and number of iterations, the value $\delta=0.5$ is a good setting regardless of the definition of the error.

A second series of tests is performed to evaluate both the effect of using symmetry (34) and of increasing the size $l$ of the space $\mathfrak{G}$ in which initial guesses are generated. Ten initialization grids are tested with error $\boldsymbol{\epsilon}_{\mathbf{2}}$, step $\delta=0.5$, five values of $l$ and with or without the symmetry. This yields 797138 inversions, and for each of the ten resulting sets $\mathcal{L}$ the rate of success is $99.9 \%$. The average time per inversion is plotted in Figure 4 As critical points $t_{P}= \pm \lambda$ prompt numerical integration instead of analytical one (see end of 2.4), they slow down the inversion. Thus the percentage of rods $t_{P}= \pm \lambda$ among tested points is also given for a better assessment of the results. As expected, size $l$ increases the time of inversion due to the search of initial guesses in a larger grid. This holds true even though the ratio of time consuming points $t_{P}= \pm \lambda$ is lowered. Only one exception is encountered between $l=2$ and $l=3$, where the influence 


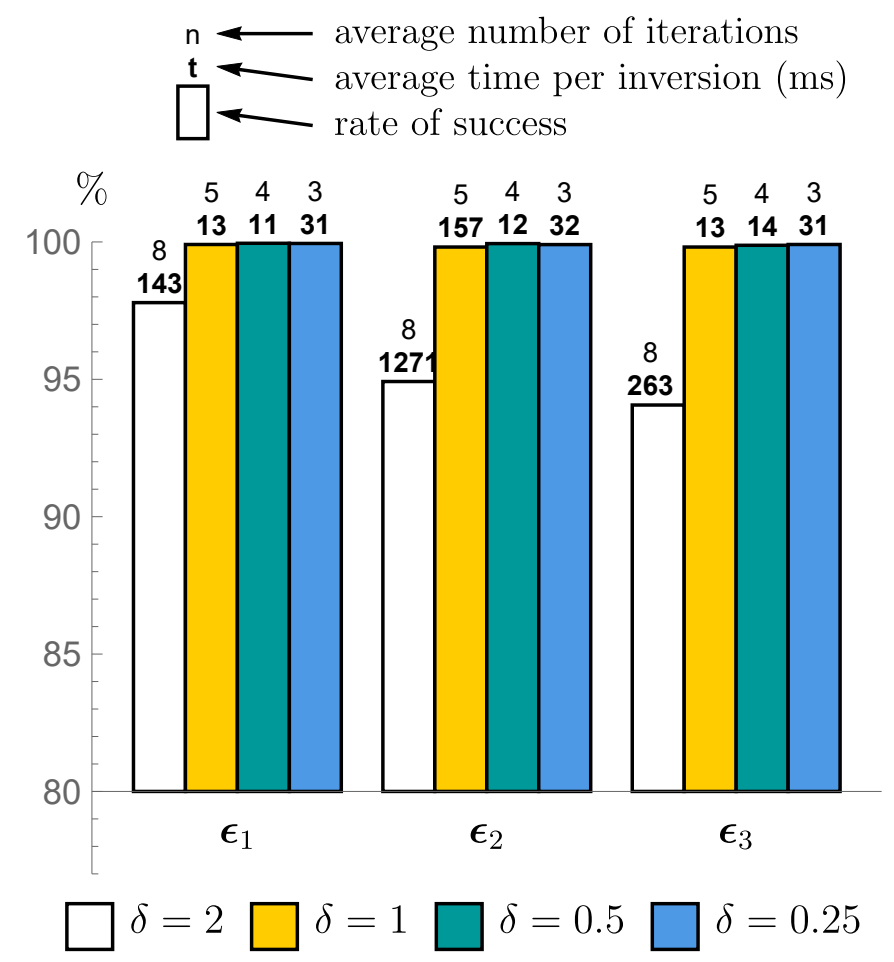

Figure 3: Rate of success, average time per inversion in ms and average number of iterations for the three error definitions proposed in 3.1 and initialization grids of same size $l=2$ and different steps $\delta=2$ ( $\alpha_{1}, 3622$ inversions), $\delta=1$ ( $\alpha_{1}, 2154$ inversions), $\delta=0.5$ ( $\alpha_{2}, 53$ 170 inversions), $\delta=0.25$ ( $\alpha_{2}, 50946$ inversions). The scaled torsional rigidity is set at $K_{30}=1$.

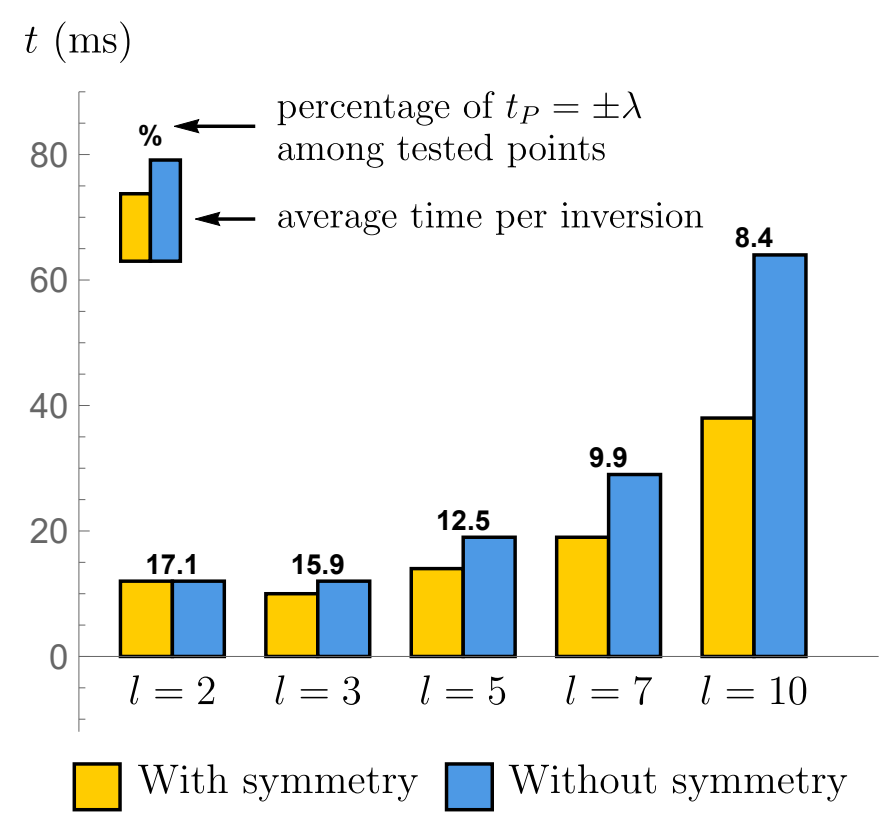

Figure 4: Average time per inversion for error $\boldsymbol{\epsilon}_{\mathbf{2}}$, step $\delta=0.5$ and different sizes $l=2(53170$ inversions), $l=3$ ( 82260 inversions), $l=5$ (142552 inversions), $l=7$ (191004 inversions), $l=10$ (328 152 inversions). Expression $\alpha_{2}$ is used for the spacing of tested points. The percentage of rod geometries $t_{P}= \pm \lambda$ among the tested points is also displayed, because these singular points have an influence on the speed of inversion. Two initialization grids are tested for each value of $l$ : the first one uses symmetry (34), but not the second one. All the ten resulting sets of inversions have a success rate of $99.9 \%$. Note that the overall 797138 inversions test distinct anchoring boundary conditions. The computations are made with a scaled torsional rigidity $K_{30}=1$.

of points $t_{P}= \pm \lambda$ is predominant. The advantage of using symmetry (34) is also well evidenced as it decreases the computation cost.

The image under $q_{1}$ of the discrete set $\mathfrak{H}_{d}$ of tested points is plotted in Figure 5 in the case where the initialization grid $\mathcal{G}$ has a size $l=2$ and a discretization step $\delta=0.5$. This shows the set of clamping parameter values that have been tested and reached. We noticed that the boundaries of this space are not changed when $l$ is increased at $l=10$ or when the length of the rod is extended up to four periods. Thus, the boundary conditions reached in this article are quite well representative of the equilibrium configurations of ideal 3D elastic rods. 


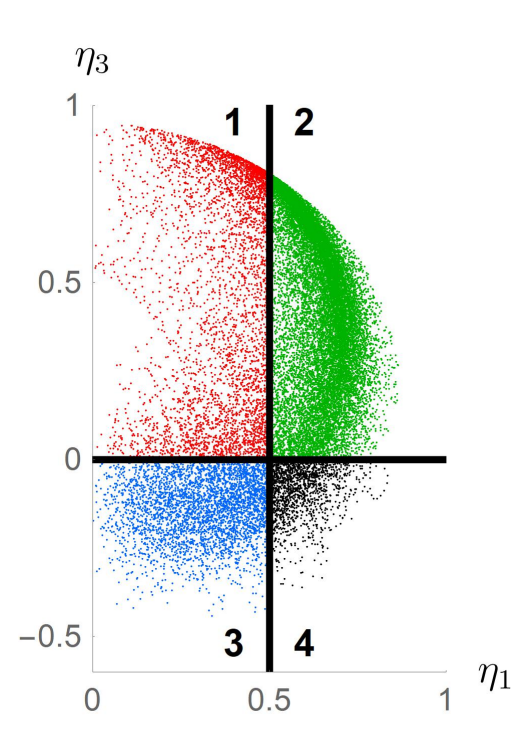

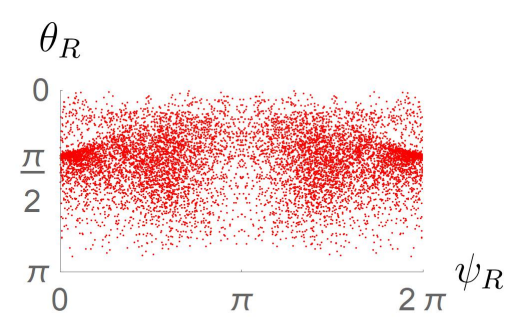

Region 1

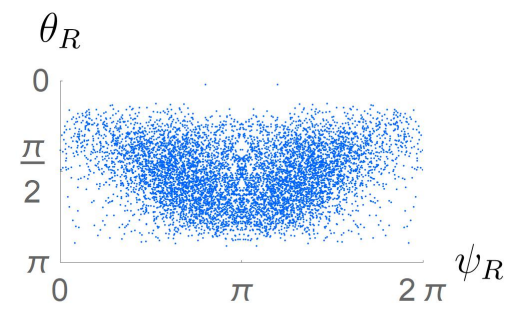

Region 3

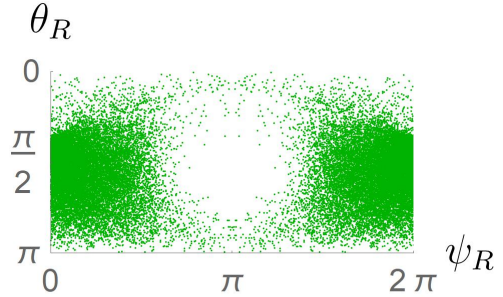

Region 2

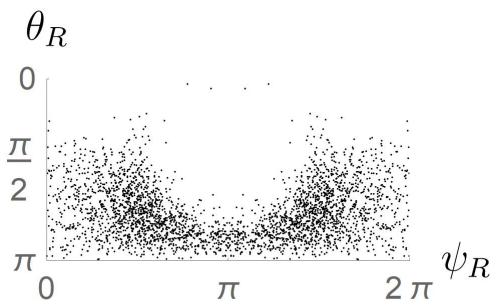

Region 4

Figure 5: Plot of the values of clamping parameters that are targeted for grid size $l=2$ and discretization step $\delta=0.5$. The observed heterogeneity results from scanning regularly the input space $\mathfrak{H}$ instead of the output space. Tests are performed only for points $\eta_{1} \geq 0$ and $\eta_{2}=0$, from which all other points can be obtained using the rotational symmetry exhibited by equations 15 and 16 . To highlight the intricate dependence of targeted Euler angles on targeted relative positions, the plot $\left(\eta_{1}, \eta_{2}\right)$ is divided in four regions and the corresponding values of $\left(\psi_{R}, \theta_{R}\right)$ are plotted separately. Because it is difficult to visualize a $5 \mathrm{D}$ space, $\varphi_{R}$ is not plotted.

\subsection{Extensions of the method}

The results of the two series of tests performed in section 4 show that our method is efficient to provide one analytical rod expression for given strong anchoring conditions. It is then possible to obtain other solutions by numerical continuation in parameters $\left(\psi_{R}, \theta_{R}, \varphi_{R}\right)$. An example is given in Figure 6, where a second solution is obtained by adding one turn on $\varphi_{R}$. The rod solutions computed with this strategy may be distinguished using the net winding $\mathcal{L}_{p}$ introduced by Berger and Prior $([38,39])$. This quantity is a generalization of the linking number to open rods bound between two parallel planes. The net winding is written as the sum of the polar writhe $\mathcal{W}_{p}$, a measure of the contortion, and of the twist $\mathcal{T}$, a measure of the total rotation of the Darboux frame around the tangent :

$$
\mathcal{L}_{p}=\mathcal{W}_{p}+\mathcal{T}
$$

Two solutions of the same boundary conditions, obtained by adding or removing turns on Euler angles $\left(\psi_{R}, \theta_{R}, \varphi_{R}\right)$, will have net windings that differ by an integer. For instance, the first solution shown in Figure 6 is such that $\mathcal{L}_{p}=0.1$, and the second one verify $\mathcal{L}_{p}=1.1$. The difference between the two net windings is one, indicating that there is exactly one more turn in the second solution. Yet, this strategy is not sufficient to compute all elastic rod shapes corresponding to given strong anchoring conditions : there may exists different solutions with the same value of net winding, as illustrated below.

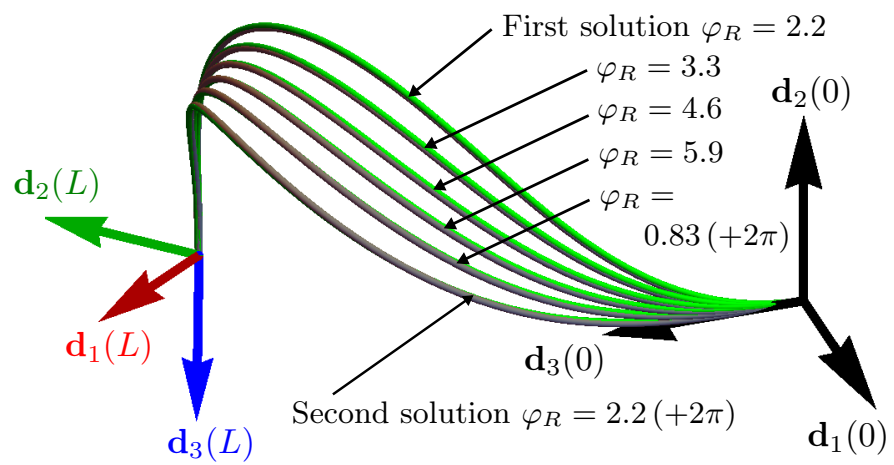

Figure 6: Example of numerical continuation in parameter $\varphi_{R}$ with $\boldsymbol{q}=\{-0.4,-0.1,0.5,4.8,1.4,2.2\}$. Starting from a first solution $\boldsymbol{p}=\{-0.3,-0.2,-0.7,3.4,0.5,4.8\}, \varphi_{R}$ is increased progressively using algorithms 1 and 2 initialized at the current elastic rod. After an entire turn, a second solution $\boldsymbol{p}=\{1.1,1.5,3.1,0.64,1.8,3.3\}$ is obtained. Rod color convention is as in legend to Figure 1 The computations are made with a scaled torsional rigidity $K_{30}=1$.

To obtain more solutions, another idea is to use several initial guesses from which Newton's method will converge towards different roots. This is relevant because each solution has potentially a large set of initial points, called its 
basin of attraction, from which it can be reached with the search algorithm. For instance, instead of taking the initial

190 points of $\mathcal{G}$ which are the closest. The value of $k$ may nonetheless be difficult to choose. The higher it is, the more solutions are to be obtained, but the computation cost is increased as more initial guesses are used. In addition, this strategy is not very efficient because many initial guesses may converge to the same solution, while others will lead to singular points in which the algorithm remains trapped. Still, it is a very simple way to obtain many solutions. It are quite particular constraints as the two end tangents are aligned. As a consequence, the result does not depends on parameter $\varphi_{0}$, whose value is set at 3 for each solution. To obtain the values of $\boldsymbol{p}_{5 D}$, the $k=180$ initial guesses of grid $\mathcal{G}$ whose clamping parameters are the closest to the targeted ones are used to initiate search algorithm 1 . Among these initial guesses, only seven lead to singular points. All others lead to solutions, but some solutions are obtained is lower than $10^{-1}$. After eliminating these duplicates, 32 solutions are obtained. By selecting only the ones with a dimensionless length $L / \mu$ smaller than three times the period $s_{\text {per }} / \mu$, ten solutions remain. Four of them are plotted in Figure 7: : they are two pairs of elastic rods with the same net winding, but different writhe and different twist. Note that the difference between the two values of net winding is an integer, thus one solution $\mathcal{L}_{p}=-1.58$ may be

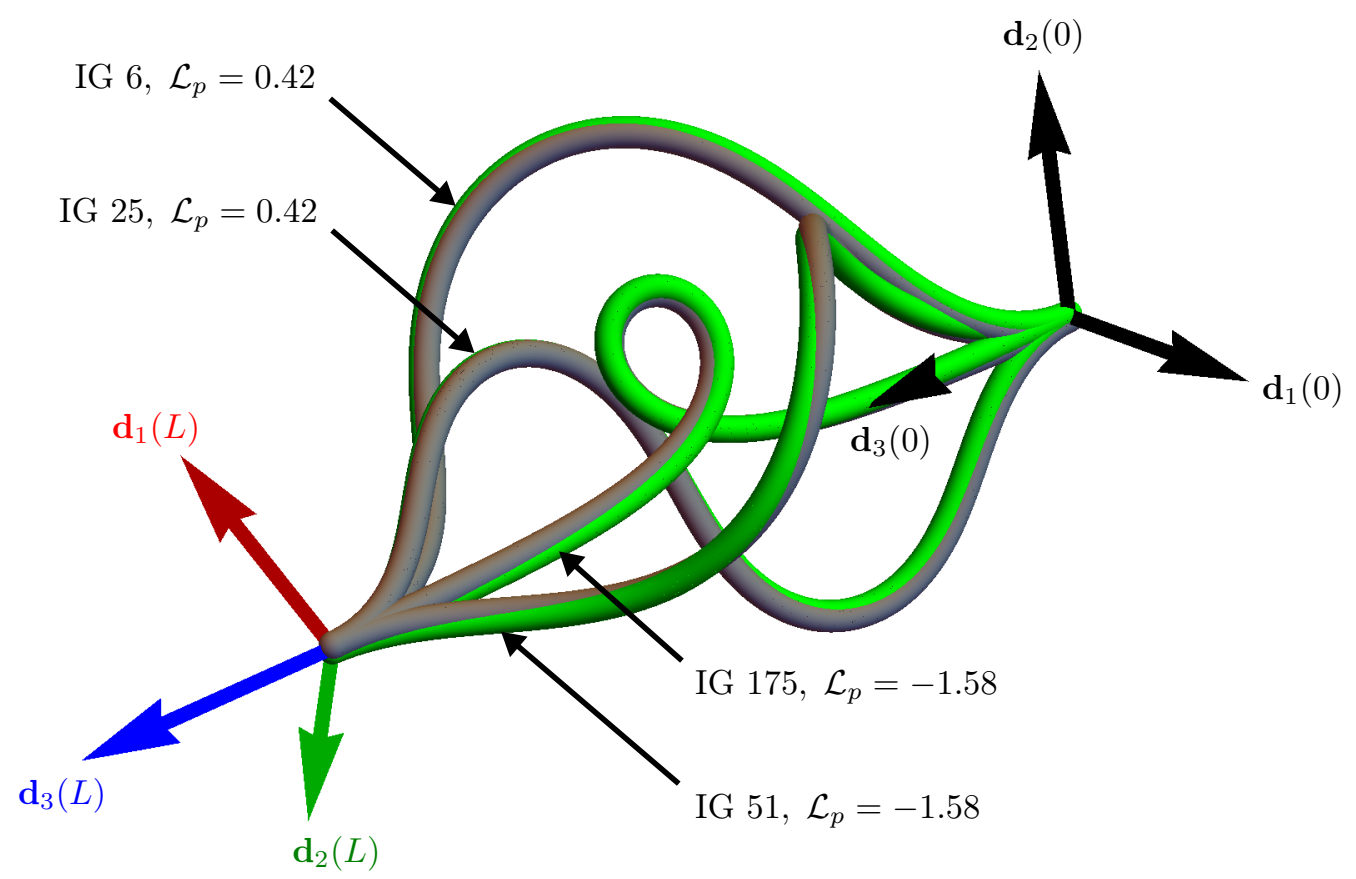

Figure 7: Example of four elastic rods that satisfy the boundary conditions $\boldsymbol{q}=\{0,0,0.7,2 \pi \times 0.42,0,0\}$, computed from four of the 180 initial guesses of grid $\mathcal{G}$ whose clamping parameters are the closest to the targeted ones, $\boldsymbol{q}$. For each solution, the rank of the initial guess (IG) used (all 180 are ordered by increasing error) and the value of the net winding $\mathcal{L}_{p}$ are indicated. The computations are made with a scaled torsional rigidity $K_{30}=0.7$. The values of physical parameters $\boldsymbol{p}_{5 D}$ are : $\{-0.4,0.2,0.8,0.2,6.4\}(\mathrm{IG} 6),\{-0.1,0.2,-0.8,0.3,9.7\}$ (IG 25), $\{0.6,-1.3,3.0,0.7,4.1\}$ (IG 51), $\{-0.2,-0.2,2.0,6.4,13.3\}$ (IG 175). Rod color convention is as in legend to Figure 1

These two strategies, whose results are shown in Figures 6 and 7 , are interesting possibilities to find many rod shapes with imposed boundary conditions. Then, the shapes can be classified using quantities as the net winding, the number of periods or the elastic energy of the rod. Further studies must be carried out to reduce the computation cost of these strategies. For example, the second one may be improved by stopping algorithm 1 as soon as the number of period is beyond a chosen limit, or when a local minimum is detected. However, an entire resolution of the boundary value problem, with all pertinent solutions determined, remains a difficult matter beyond the scope of this paper.

\section{Conclusion}

A general approach is presented to compute the equilibrium configurations of ideal 3D elastic rods with specified strong anchoring boundary conditions, i.e. end-to-end relative position and orientation of the Darboux frame attached sections. This translates into an inverse problem that we have formulated with two forward functions, $q_{1}$ and $q_{2}$ whose output space is the set of all possible boundary conditions. They have been expressed analytically. On the first hand, the 5D function $q_{1}(\boldsymbol{p})$ is derived from L.D. Landau and E.M. Lifshitz [31] and possesses symmetry properties. With these properties, the problem has been restricted from six to five dimensions and the work space has been reduced to $t_{P} \geq 0$. Initial guesses have been provided efficiently by constructing an initialization grid in the five-dimensional 
input space of $q_{1}$. This grid consists in a set of values of physical parameters $\boldsymbol{p}$ associated to their image under $q_{1}$. In a broad perspective, these initial guesses are required whatever numerical method is developed (root-finding, continuation or finite elements). On the other hand, the $6 \mathrm{D}$ function $q_{2}(\boldsymbol{f})$ is particularly suited to numerical methods as it is defined in all $\mathbb{R}^{6}$. This is why it has been implemented in Newton-Raphson's algorithm.

The two performed series of tests amount to more than 850000 inversions. In $99.9 \%$ of cases, the targeted boundary conditions have been attained with a minimum precision of $10^{-5}$. This rate of success has been obtained for all three tested definitions of the error. The solutions are formulated through values of physical parameters $\boldsymbol{p}$, which gives an entire geometric and mechanical description of the rod configuration in analytical form. We have obtained an optimal value of the discretization step of the initialization grid, $\delta=0.5$, which maximizes the success rate and minimizes the computational cost. For $\delta=0.5$, the success rate remains constant when increasing the characteristic size $l$ of the exploration space from $l=2$ to $l=10$. The augmentation of computational time due to larger initialization grids has been plotted.

The strategy we have proposed can be improved in several manners. First, a detailed analysis of function $q_{1}$ may help estimate the region of input space where the solutions are and restrict the size of the initialization grid. Especially, the classifications obtained in [30 may be used. Second, the proposed extensions to obtain many other solutions should be tested on more examples and rendered more efficient. In particular, the inversion could be enhanced by using more robust root-finding methods. Criteria must be defined to identify irrelevant initial guesses. Statistical studies of the initialization grid would probably help select relevant ones. The work proposed here is a serious move forward in the resolution of this difficult two-point boundary value problem. Some novel ideas exposed will give rise to further developments.

\section{Acknowledgements}

The authors acknowledge support from the program "Convergence UPMC" and the PhD program "Interface Pour le Vivant UPMC". They especially thank S. Neukirch for helpful discussions.

\section{References}

[1] J. F. Marko, S. Neukirch, Competition between curls and plectonemes near the buckling transition of stretched supercoiled DNA, Phys. Rev. E 85 (1) (2012) 011908. doi:10.1103/PhysRevE.85.011908

口[ [2] T. McMillen, P. Holmes, An elastic rod model for anguilliform swimming, J. Math. Biol. 53 (5) (2006) 843-886. doi:10.1007/ s00285-006-0036-8

[3] F. Dal Corso, D. Misseroni, N. Pugno, A. Movchan, N. Movchan, D. Bigoni, Serpentine locomotion through elastic energy release, J. R. Soc. Interface 14 (130) (2017) 20170055. doi:10.1098/rsif.2017.0055

[4] V. G. A. Goss, R. Chaouki, Loading paths for an elastic rod in contact with a flat inclined surface, Int. J. Solids Struct. 88 (2016) 274-282. doi:10.1016/j.ijsolstr.2016.02.042

[5] L. N. Claverie, Y. Boubenec, G. Debrégeas, A. M. Prevost, E. Wandersman, Whisker contact detection of rodents based on slow and fast mechanical inputs, Front. Behav. Neurosci. 10. doi:10.3389/fnbeh.2016.00251

[6] L. B. Kratchman, T. L. Bruns, J. J. Abbott, R. J. Webster, Guiding elastic rods with a robot-manipulated magnet for medical applications, IEEE T. Robot. 33 (1) (2017) 227-233. doi:10.1109/TRO.2016.2623339.

[7] J. Fang, J.-S. Chen, Deformation and vibration of a spatial elastica with fixed end slopes, Int. J. Solids Struct. 50 (5) (2013) $824-831$. doi:10.1016/j.ijsolstr.2012.11.011

[8] S. Goyal, N. C. Perkins, C. L. Lee, Nonlinear dynamics and loop formation in Kirchhoff rods with implications to the mechanics of DNA and cables, J. Comput. Phys. 209 (1) (2005) 371-389. doi:10.1016/j.jcp.2005.03.027

[9] C. Pakleza, J. A. H. Cognet, Biopolymer Chain Elasticity: a novel concept and a least deformation energy principle predicts backbone and overall folding of DNA TTT hairpins in agreement with NMR distances, Nucleic Acids Res. 31 (3) (2003) 1075-1085. doi: $10.1093 / \mathrm{nar} / \mathrm{gkg} 194$

[10] G. P. Santini, C. Pakleza, J. A. H. Cognet, DNA tri-and tetra-loops and RNA tetra-loops hairpins fold as elastic biopolymer chains in agreement with PDB coordinates, Nucleic Acids Res. 31 (3) (2003) 1086-1096. doi:10.1093/nar/gkg196

[11] G. P. Santini, J. A. H. Cognet, D. Xu, K. K. Singarapu, C. Hervé du Penhoat, Nucleic acid folding determined by mesoscale modeling and NMR spectroscopy: solution structure of d(GC GAAA GC), J. Phys. Chem. B 113 (19) (2009) 6881-6893. doi:10.1021/jp8100656

[12] M. Baouendi, J. A. H. Cognet, C. S. Ferreira, S. Missailidis, J. Coutant, M. Piotto, E. Hantz, C. Hervé du Penhoat, Solution structure of a truncated anti-MUC1 DNA aptamer determined by mesoscale modeling and NMR, FEBS J. 279 (3) (2012) 479-490. doi:10.1111/j.1742-4658.2011.08440.x

[13] S. S. Antman, Nonlinear Problems of Elasticity, 2nd Edition, Applied Mathematical Sciences, Springer, New York, 2005. doi: $10.1007 / 0-387-27649-1$

[14] H. Tsuru, Equilibrium shapes and vibrations of thin elastic rod, J. Phys. Soc. Jpn. 56 (7) (1987) 2309-2324. doi:10.1143/JPSJ .56.2309

[15] Y. Shi, J. E. Hearst, The Kirchhoff elastic rod, the nonlinear Schrödinger equation, and DNA supercoiling, J. Chem. Phys. 101 (6) (1994) 5186-5200. doi:10.1063/1.468506

[16] I. Tobias, B. D. Coleman, W. K. Olson, The dependence of DNA tertiary structure on end conditions: theory and implications for topological transitions, J. Chem. Phys. 101 (12) (1994) 10990-10996. doi:10.1063/1.467849

[17] J. Langer, D. A. Singer, Lagrangian aspects of the Kirchhoff elastic rod, SIAM Rev. 38 (4) (1996) 605-618. doi:10.1137/ S0036144593253290

[18] D. J. Dichmann, Y. Li, J. H. Maddocks, Hamiltonian formulations and symmetries in rod mechanics, in: Mathematical approaches to biomolecular structure and dynamics, Springer, New York, 1996, pp. 71-113. doi:10.1007/978-1-4612-4066-2_6

[19] M. Nizette, A. Goriely, Towards a classification of Euler-Kirchhoff filaments, J. Math. Phys. 40 (6) (1999) 2830-2866. doi:10.1063/ 1.532731 .

[20] J. H. Maddocks, R. S. Manning, R. C. Paffenroth, K. A. Rogers, J. A. Warner, Interactive computation, parameter continuation, and visualization, Int. J. Bifurcat. Chaos 7 (08) (1997) 1699-1715. doi:10.1142/S0218127497001333. 
[21] A. Balaeff, L. Mahadevan, K. Schulten, Elastic rod model of a DNA loop in the Lac operon, Phys. Rev. Lett. 83 (1999) $4900-4903$. doi:10.1103/PhysRevLett.83.4900

[22] B. D. Coleman, D. Swigon, Theory of supercoiled elastic rings with self-contact and its application to DNA plasmids, J. Elasticity 60 (3) (2000) 173-221. doi:10.1023/A:1010911113919

[23] G. H. M. Van der Heijden, S. Neukirch, V. G. A. Goss, J. Thompson, Instability and self-contact phenomena in the writhing of clamped rods 45 (1) (2003) 161-196. doi:10.1016/S0020-7403(02)00183-2

[24] M. E. Henderson, S. Neukirch, Classification of the spatial equilibria of the clamped elastica: numerical continuation of the solution set, Int. J. Bifurcat. Chaos 14 (04) (2004) 1223-1239. doi:10.1142/S0218127404009971

[25] M. Bergou, M. Wardetzky, S. Robinson, B. Audoly, E. Grinspun, Discrete elastic rods, in: ACM T. Graphics, Vol. 27, ACM, 2008, p. 63. doi:10.1145/1360612.1360662

[26] A. Lazarus, J. Miller, P. Reis, Continuation of equilibria and stability of slender elastic rods using an asymptotic numerical method, J. Mech. Phys. Solids 61 (8) (2013) 1712-1736. doi:10.1016/j.jmps.2013.04.002

[27] R. S. Manning, A catalogue of stable equilibria of planar extensible or inextensible elastic rods for all possible Dirichlet boundary conditions, J. Elasticity 115 (2) (2014) 105-130. doi:10.1007/s10659-013-9449-y

[28] T. Bretl, Z. McCarthy, Quasi-static manipulation of a Kirchhoff elastic rod based on a geometric analysis of equilibrium configurations, Int. J. Robot Res. 33 (1) (2014) 48-68. doi:10.1177/0278364912473169

[29] B. D. Coleman, I. Tobias, D. Swigon, Theory of the influence of end conditions on self-contact in DNA loops, J. Chem. Phys. 103 (20) (1995) 9101-9109. doi:10.1063/1.470021

[30] O. Ameline, S. Haliyo, X. Huang, J. A. H. Cognet, Classifications of ideal 3D elastica shapes at equilibrium, J. Math. Phys. 58 (6) (2017) 062902. doi:10.1063/1.4989556.

[31] L. D. Landau, E. M. Lifshitz, A. M. Kosevich, L. P. Pitaevskiř, Theory of Elasticity, 3rd Edition, no. 7 in Course of theoretical physics, Pergamon Press, 1986.

URL https://books.google.fr/books?id=tpY-VkwCkAIC

[32] J. Stoer, R. Bulirsch, Introduction to Numerical Analysis, 3rd Edition, Vol. 12, Springer, New York, 2002. doi:10.1007/ 978-0-387-21738-3

[33] W. Press, Numerical Recipes 3rd Edition: The Art of Scientific Computing, Cambridge University Press, 2007. URL https://books .google.fr/books?id=1aAOdzK3FegC

[34] C. Ridders, Accurate computation of $F^{\prime}(x)$ and $F^{\prime}(x) F^{\prime \prime}(x)$, Adv. Eng. Softw. 4 (2) (1982) 75-76. doi:10.1016/S0141-1195(82) $80057-0$

[35] J.-F. Bonnans, J. C. Gilbert, C. Lemaréchal, C. A. Sagastizábal, Numerical optimization: theoretical and practical aspects, 2nd Edition, Springer Science \& Business Media, Berlin Heidelberg, 2006. doi:10.1007/978-3-540-35447-5

[36] Y. N. Dauphin, R. Pascanu, C. Gulcehre, K. Cho, S. Ganguli, Y. Bengio, Identifying and attacking the saddle point problem in high-dimensional non-convex optimization, in: Adv. Neur. In., 2014, pp. 2933-2941.

[37] R. Ge, F. Huang, C. Jin, Y. Yuan, Escaping from saddle points - online stochastic gradient for tensor decomposition, in: COLT, 2015, pp. 797-842. URL http://proceedings.mlr.press/v40/Ge15.pdf

[38] M. A. Berger, C. Prior, The writhe of open and closed curves, J. Phys. A-Math. Gen. 39 (26) (2006) 8321. doi:10.1088/0305-4470/ $39 / 26 / 005$

[39] C. B. Prior, S. Neukirch, The extended polar writhe: a tool for open curves mechanics, J. Phys. A-Math. Gen. 49 (21) (2016) 215201. doi: $10.1088 / 1751-8113 / 49 / 21 / 215201$. 